\title{
Hiding Messages into Secure Connection Transmissions with Full-Duplex Receiver
}

This paper was downloaded from TechRxiv (https://www.techrxiv.org).

LICENSE

CC BY 4.0

SUBMISSION DATE / POSTED DATE

$19-10-2021 / 21-10-2021$

CITATION

Ta, Hien; Nguyen, Lap Luat; Nguyen, Tien Tung; Fiche, Anthony; Gautier, Roland (2021): Hiding Messages into Secure Connection Transmissions with Full-Duplex Receiver. TechRxiv. Preprint.

https://doi.org/10.36227/techrxiv.16832110.v1

$\mathrm{DOI}$

10.36227/techrxiv.16832110.v1 


\title{
Hiding Messages into Secure Connection Transmissions with Full-Duplex Receiver
}

\author{
Lap Luat Nguyen, Tien-Tung Nguyen, Anthony Fiche, Roland Gautier, and Hien Q. Ta
}

\begin{abstract}
This letter considers hiding messages into overt transmissions with a Full-Duplex receiver, which emits Artificial Noise to secure its transmission connection while a transmitter opportunistically sends a covert message to a covert user. The warden's uncertainties of decoding the overt message and Artificial Noise received power are exploited to hide messages. Then, the covert throughput accompanied with the warden's average detection error probability are determined. The results show that increasing the transmit power of Artificial Noise will improve the covert performance. The results also show that the covert performance is improved when the self-interference cancellation is improved at the Full-Duplex receiver or the warden is located close to the Full-Duplex receiver, indicating the positive impact of the overt performance on the covert performance.
\end{abstract}

\section{INTRODUCTION}

In wireless transmissions, the security and privacy of their broadcast nature becomes a critical issue as it operates not only in civil area but also in the military. For example, privacy information in health care, journey or location of vehicles in transportation and position or confidential information of the targets need to protect. Regarding to security protections for wireless communications, several conventional approaches such as cryptography, [1] or physical layer security [2] have been implemented. These methods only target to secure confidential content of sending message from being stolen by eavesdroppers. Nevertheless, some scenarios dedicate to avoid being attacked such as jamming, when the existence (privacy) of transmissions is revealed. For example of self-driving car, the controlling signals need to be completely secured or hidden from the adversary. Moreover, the location or itinerary of vehicles needs to be kept private. Their revelations to attacker may cause security issues or accidents. Therefore, covert communication has emerged as a potential solution for dealing with the issue of privacy.

In general, two well-known approaches adopted for covert communications have been widely investigated in the literature. The key idea of the first approach is to take advantage of various sources of the adversary's uncertainty to guarantee the covertness. By exploiting the adversary's noise uncertainty in [3], a non-zero covert rate could be obtained. Later, the noise uncertainty has been extended to uninformed jamming [4], and to Artificial Noise (AN) generated by a receiver in

Lap Luat Nguyen and Hien Q. Ta are with School of Electrical Engineering, International University, Ho Chi Minh City, Vietnam and Vietnam National University, Ho Chi Minh City, Vietnam (email: nlluat@hcmiu.edu.vn, tqhien@hcmiu.edu.vn).

Tien-Tung Nguyen is with Telecommunication Division, Industrial University of Ho Chi Minh City, Ho Chi Minh City 700000, Vietnam (email: nguyentientung@iuh.edu.vn).

Anthony Fiche and Roland Gautier are with Univ Brest, Lab-STICC, CNRS, UMR 6285, F-29200, Brest, France (email: anthony.fiche@univbrest.fr, roland.gautier@univ-brest.fr).
Full-Duplex (FD) mode [5], [6]. Many other uncertainties such as transmission time [7], channel state information [8] and transmit power [9] have also been exploited for the covertness. For the second approach, other works considered superimposing the covert message into another message of existing transmissions, termed as hiding messages into overt transmissions, and exploiting the channel variations [10], controlling transmit power [11] and random transmit power [12].

The existing FD receiver schemes in [5] and [6] considered the covert receiver generating the $\mathrm{AN}$, which may cause the existence of covert transmissions being detected. Therefore, we introduce a novel scheme of hiding messages into existing overt transmissions with a FD overt receiver. More specifically, the superposition signal of the covert and overt messages is transmitted at a fixed transmit power while the overt receiver emits the AN to secure its connection. With this setting, we find that, to detect the covert message, it is necessary for the warden to decode the overt message and, even if the overt message is decoded, the covertness still can be guaranteed due to the adversary's uncertainty of receiving AN powers. In this paper, the adversary's average detection error probability as well as the covert throughput will be determined. Moreover, the AN is efficient to improve the covertness will be shown. The improved performance of the overt transmissions can help improve the covert performance will also be shown.

\section{SySTEM MODEL}

We consider one transmitter (Alice) and two receivers one (Carol) to receive overt messages, $\mathbf{v}=\left(v_{1}, \ldots, v_{n}\right)$, and one $(\mathrm{Bob})$ to receive covert messages, $\mathbf{u}=\left(u_{1}, \ldots, u_{n}\right)$; and a warden (Willie) detecting the covert transmission. The proposed system is illustrated in Fig. 1, where Carol equipped with two antennas operates at FD mode while other nodes employed Half-Duplex mode with single antenna. While one antenna of Carol is to receive its message from Alice, another is to transmit the AN to secure its connection to the warden [13]. Here, the AN is also used to hide the covert message to Bob.

Construction: the codewords $\mathbf{u}$ and $\mathbf{v}$ are independently generated by random selection symbols from a complex normal distribution. To guarantee the covertness, the codebook of $\mathbf{u}$ is a share secret between Alice and Bob while that of $\mathbf{v}$ is assumed known to all users including Willie.

\section{A. Transmitted Signal}

The transmitted signal from Alice, from Willie's perspective, is given by

$$
\mathbf{x}= \begin{cases}\sqrt{P_{a}} \mathbf{v}, & H_{0}, \\ \sqrt{P_{a}}(\sqrt{\alpha} \mathbf{v}+\sqrt{1-\alpha} \mathbf{u}), & H_{1},\end{cases}
$$




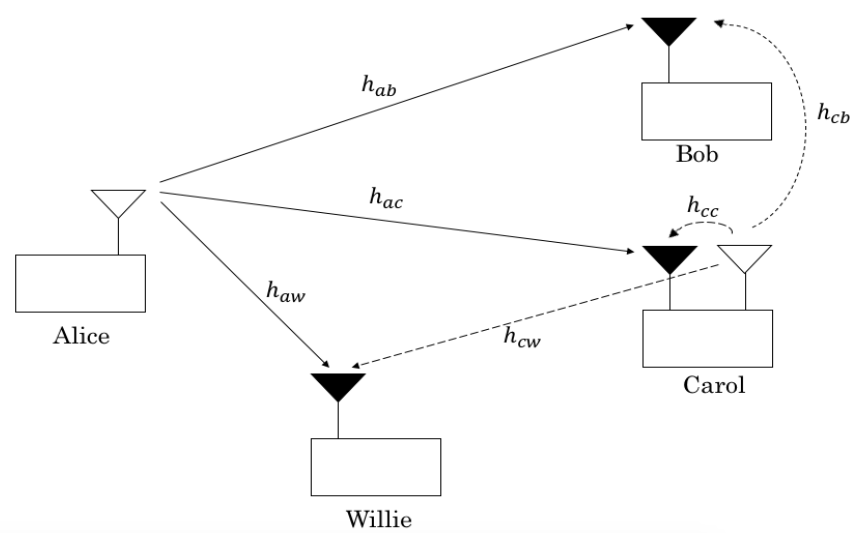

Fig. 1. Alice tries to hide a covert message to Bob within overt transmissions to Carol toward Willie, a warden looks for covert message.

where $H_{0}$ and $H_{1}$ denote the null hypothesis that $\mathbf{u}$ is not been sent by Alice and the alternative hypothesis, respectively, $\alpha \in$ $[0,1]$ is the ratio of power allocated to the overt message and $P_{a}$ is Alice's total transmit power, which is always constant whatever the covert transmission.

The quasi-static Rayleigh block fading channels is considered, where the channel gain is constant inside a $n$ symbols block and changes from one block to another independently. Let the channel gain, $h_{i j}$ between nodes $i$ and $j$, where $i \in\{a, c\}$ and $j \in\{b, c, w\}$, in which nodes $a, b, c$ and $w$ represent Alice, Bob, Carol and Willie, respectively, and have Gaussian distribution with mean 0 and variance $\sigma_{i j}^{2}$. Assuming that Alice sends pilot symbols for channel estimations before data transmissions. Assuming perfect channel estimation at all receiver nodes, node $j$ perfectly knows $h_{a j}$. We further denote $\mathbf{n}_{j}, j \in\{b, c, w\}$, as the background noise vector at node $j$ and assume that $\mathbf{n}_{j}$ has complex Gaussian distribution with mean 0 and variance $\sigma_{n}^{2}$, i.e. $\mathbf{n}_{j} \sim C N\left(0, \sigma_{n}^{2}\right)$.

\section{B. Overt Transmission Rate}

Under $H_{1}$, the received signal at Carol is given by

$$
\boldsymbol{y}_{c}=\sqrt{\alpha P_{a}} h_{a c} \mathbf{v}+\sqrt{(1-\alpha) P_{a}} h_{a c} \mathbf{u}+\sqrt{\phi P_{c}} h_{c c} \mathbf{z}+\mathbf{n}_{c},
$$

where $\mathbf{z} \sim C N(0,1)$ is the AN signal transmitted by Carol and $P_{c}$ is the transmit AN power. Although the AN is known to Carol, it cannot be absolutely cancelled and, in practical, can be eliminated with a cancellation coefficient, $\phi$, where $0<\phi \leq 1$ [14]. Different from [5] where the random transmit power of the AN is considered to provide Willie's uncertainty, this paper considers an AN's fixed transmit power, which is publicly known to all nodes including Willie. However, since there is no pilot symbols sent by Carol and it is impossible for Willie to estimate the channel gain $h_{c w}$ with the unknown AN, Willie does not know $h_{c w}$ and hence, has uncertainty of his received AN power, $\left|h_{c w}\right|^{2} P_{c}$.

Since Carol does not know the presence of $\mathbf{u}$, the capacity of $\mathbf{v}$ is given by

$$
I\left(\mathbf{v} ; \mathbf{y}_{c}\right)=\log _{2}\left(1+\frac{\alpha\left|h_{a c}\right|^{2} P_{a}}{\sigma_{n}^{2}+(1-\alpha)\left|h_{a c}\right|^{2} P_{a}+\phi\left|h_{c c}\right|^{2} P_{c}}\right) \text {. }
$$

Alice considers transmitting the overt message at a fixed rate $R_{v}$ due to unknown $h_{a c}$. Since $\left|h_{i j}\right|^{2}$ is distributed exponentially with a scale of $1 / \sigma_{i j}^{2}$, the probability of overt decoding outage probability is obtained by

$$
\begin{aligned}
P_{\text {out }, C} & =\operatorname{Pr}\left(I\left(\mathbf{v} ; \mathbf{y}_{c}\right)<R_{v}\right) \\
& =\operatorname{Pr}\left(\left|h_{a c}\right|^{2}<\frac{\left(2^{R_{v}}-1\right)\left(\sigma_{n}^{2}+\phi\left|h_{c c}\right|^{2} P_{c}\right)}{\left(1-(1-\alpha) 2^{R_{v}}\right) P_{a}}\right) \\
& =1-\frac{\exp \left(-\frac{\left(2^{R_{v}}-1\right) \sigma_{n}^{2}}{\left(1-(1-\alpha) 2^{R_{v}}\right) P_{a} \sigma_{a c}^{2}}\right)}{1+\frac{\left(2^{R_{v}}-1\right) \phi P_{c} \sigma_{c c}^{2}}{\left(1-(1-\alpha) 2^{R_{v}}\right) P_{a} \sigma_{a c}^{2}}} .
\end{aligned}
$$

We consider transmitting the overt message at the rate $R_{v}$ to maximize the overt throughput,

$$
\eta_{v}=R_{v} \times\left(1-P_{\text {out }, C}\right),
$$

where $R_{v}$ can be found by numerical search.

\section{WARDEN's OPTIMUM DETECTION}

The signal to be received at Willie is expressed as

$\mathbf{y}_{w}= \begin{cases}\sqrt{P_{a}} h_{a w} \mathbf{v}+\sqrt{P_{c}} h_{c w} \mathbf{z}+\mathbf{n}_{w}, & H_{0}, \\ \sqrt{P_{a}} h_{a w}(\sqrt{\alpha} \mathbf{v}+\sqrt{1-\alpha} \mathbf{u})+\sqrt{P_{c}} h_{c w} \mathbf{z}+\mathbf{n}_{w}, & H_{1} .\end{cases}$

It will be proved that the distribution of $\mathbf{y}_{w}$ is identical under $H_{0}$ and $H_{1}$ if $\mathbf{v}$ is not decoded, and different if $\mathbf{v}$ is decoded. This means that Willie cannot detect the covert message $\mathbf{u}$ if the overt message $\mathbf{v}$ cannot be decoded. Moreover, since Willie has uncertainty of the received AN power, $\left|h_{c w}\right|^{2} P_{c}$, the covertness can still be guaranteed if $\mathbf{v}$ is decoded. In detail, we consider two cases: Willie fails to decode $\mathbf{v}$ and Willie succeeds in decoding $\mathbf{v}$, as follows.

\section{A. Willie fails to decode $\mathbf{v}$}

When Willie fails to decode $\mathbf{v}$, he will perform the marginalized likelihood ratio test (LRT), i.e. averaging unknown $\mathbf{v}$ in the likelihood functions, as its optimum detection [15],

$$
\Lambda:=\frac{\mathrm{E}_{\mathbf{v}}\left[\operatorname{Pr}\left(\mathbf{y}_{w} \mid \mathbf{v}, H_{1}\right)\right]}{\mathrm{E}_{\mathbf{v}}\left[\operatorname{Pr}\left(\mathbf{y}_{w} \mid \mathbf{v}, H_{0}\right)\right]} \underset{H_{1}}{\stackrel{H_{0}}{\lessgtr}} \lambda,
$$

where $^{1}$

$$
\begin{aligned}
& \operatorname{Pr}\left(\mathbf{y}_{w} \mid \mathbf{v}, H_{1}\right)=\frac{\exp \left(-\frac{\left\|y_{w}-h_{a w} \sqrt{\alpha P_{a}} \mathbf{v}\right\|^{2}}{\sigma_{n}^{2}+\left|h_{c w}\right|^{2} P_{c}+(1-\alpha) P_{a}\left|h_{a w}\right|^{2}}\right)}{\left(\pi\left(\sigma_{n}^{2}+\left|h_{c w}\right|^{2} P_{c}+(1-\alpha)\left|h_{a w}\right|^{2} P_{a}\right)\right)^{n}} \\
& \operatorname{Pr}\left(\mathbf{y}_{w} \mid \mathbf{v}, H_{0}\right)=\frac{\exp \left(-\frac{\| y_{w}-\left.h_{a w} \sqrt{P_{a}} \mathbf{v}\right|^{2}}{\sigma_{n}^{2}+\left|h_{c w}\right|^{2} P_{c}}\right)}{\left(\pi\left(\sigma_{n}^{2}+\left|h_{c w}\right|^{2} P_{c}\right)\right)^{n}}
\end{aligned}
$$

which yields $\mathrm{E}_{\mathbf{v}}\left[\operatorname{Pr}\left(\mathbf{y}_{w} \mid \mathbf{v}, H_{1}\right)\right]=\mathrm{E}_{\mathbf{v}}\left[\operatorname{Pr}\left(\mathbf{y}_{w} \mid \mathbf{v}, H_{0}\right)\right]$. Hence, $\Lambda=1$. Then, the false alarm and missed detection probabilities, in the case that Willie fails to decode $\mathbf{v}$, are given by

$$
\begin{gathered}
P_{f}=\operatorname{Pr}\left(\Lambda>\lambda, I\left(\mathbf{v} ; \mathbf{y}_{w}\right)<R_{v} \mid H_{0}\right), \\
P_{m}=\operatorname{Pr}\left(\Lambda \leq \lambda, I\left(\mathbf{v} ; \mathbf{y}_{w}\right)<R_{v} \mid H_{1}\right)
\end{gathered}
$$

\footnotetext{
${ }^{1}$ Since Willie does not know the codebook of $\mathbf{u}, \mathbf{u}$ in (6) is treated as noise
} 
respectively, where

$I\left(\mathbf{v} ; \mathbf{y}_{w}\right)= \begin{cases}\log _{2}\left(1+\frac{\left|h_{a w}\right|^{2} P_{a}}{\sigma_{n}^{2}+\left|h_{c w}\right|^{2} P_{c}}\right), & H_{0}, \\ \log _{2}\left(1+\frac{\alpha\left|h_{a w}\right|^{2} P_{a}}{\sigma_{n}^{2}+(1-\alpha)\left|h_{a w}\right|^{2} P_{a}+\left|h_{c w}\right|^{2} P_{c}}\right), & H_{1},\end{cases}$

is the mutual information between $\mathbf{v}$ and $\mathbf{y}_{w}$, and $I\left(\mathbf{v} ; \mathbf{y}_{w}\right)<$ $R_{v}$ denotes the event of Willie's failure to decode $\mathbf{v}$.

Minimizing $P_{f}+P_{m}$ is the goal of Willie, by choosing $\lambda$ properly. Since $I\left(\mathbf{v} ; \mathbf{y}_{w}\right)$ under $H_{0}$ is larger than that under $H_{1}$, Willie may choose $\lambda \geq \Lambda$ to obtain

$$
\begin{aligned}
P_{f}+P_{m} & =\operatorname{Pr}\left(I\left(\mathbf{v} ; \mathbf{y}_{w}\right)<R_{v} \mid H_{0}\right) \\
& =\operatorname{Pr}\left(\left|h_{a w}\right|^{2}<\frac{\left(2^{R_{v}}-1\right)\left(\sigma_{n}^{2}+\left|h_{c w}\right|^{2} P_{c}\right)}{P_{a}}\right) \\
& =1-\frac{\exp \left(-\left(2^{R_{v}}-1\right) \sigma_{n}^{2} /\left(\sigma_{a w}^{2} P_{a}\right)\right)}{1+\left(2^{R_{v}}-1\right) \sigma_{c w}^{2} P_{c} /\left(\sigma_{a w}^{2} P_{a}\right)} .
\end{aligned}
$$

\section{B. Willie succeeds in decoding $\mathbf{v}$}

When Willie succeeds in decoding $\mathbf{v}$, he will perform the LRT of

$$
\begin{aligned}
\Lambda^{\prime} & :=\frac{1}{n} \ln \left(\frac{\operatorname{Pr}\left(\mathbf{y}_{w} \mid \mathbf{v}, H_{1}\right)}{\operatorname{Pr}\left(\mathbf{y}_{w} \mid \mathbf{v}, H_{0}\right)}\right)-\ln \left(\frac{\sigma_{n^{\prime}}^{2}}{\sigma_{n^{\prime}}^{2}+(1-\alpha)\left|h_{a w}\right|^{2} P_{a}}\right) \\
& =\frac{\left\|\mathbf{y}_{w}-h_{a w} \mathbf{v}\right\|^{2}}{n \sigma_{n^{\prime}}^{2}}-\frac{\left\|\mathbf{y}_{w}-h_{a w} \sqrt{\alpha} \mathbf{v}\right\|^{2}}{n\left(\sigma_{n^{\prime}}^{2}+(1-\alpha)\left|h_{a w}\right|^{2} P_{a}\right)} \sum_{H_{1}}^{H_{0}} \lambda^{\prime} .(12)
\end{aligned}
$$

As $n \rightarrow \infty, \Lambda^{\prime}$ converges to

$$
\Lambda^{\prime} \rightarrow \begin{cases}\frac{2 \sqrt{\alpha}(1-\sqrt{\alpha})\left|h_{a w}\right|^{2} P_{a}}{\sigma_{n}^{2}+\left|h_{c w}\right|^{2} P_{c}+(1-\alpha)\left|h_{a w}\right|^{2} P_{a}}, & H_{0}, \\ \frac{2(1-\sqrt{\alpha})\left|h_{a w}\right|^{2} P_{a}}{\sigma_{n}^{2}+\left|h_{c w}\right|^{2} P_{c}}, & H_{1} .\end{cases}
$$

Since Willie knows $h_{a w}$ and does not know $h_{c w}$, the false alarm and missed detection probabilities for decoding failure of $\mathbf{v}$ at Willie are given by

$$
\begin{aligned}
P_{f}^{\prime}\left(h_{a w}\right) & =\operatorname{Pr}\left(\Lambda^{\prime}>\lambda^{\prime}, I\left(\mathbf{v} ; \mathbf{y}_{w}\right) \geq R_{v} \mid H_{0}\right) \\
& =\operatorname{Pr}\left(\left|h_{c w}\right|^{2} / \sigma_{c w}^{2}<\min \left\{r_{0} / \lambda^{\prime}-s_{0}, \delta_{0}\right\}\right) \\
& =1-\exp \left(-\min \left\{r_{0} / \lambda^{\prime}-s_{0}, \delta_{0}\right\}\right), \\
P_{m}^{\prime}\left(h_{a w}\right) & =\operatorname{Pr}\left(\Lambda^{\prime} \leq \lambda^{\prime}, I\left(\mathbf{v} ; \mathbf{y}_{w}\right) \geq R_{v} \mid H_{1}\right) \\
& =\operatorname{Pr}\left(r_{1} / \lambda^{\prime}-s_{1} \leq\left|h_{c w}\right|^{2} / \sigma_{c w}^{2} \leq \delta_{1}\right) \\
& =\left(\exp \left(-\left(r_{1} / \lambda^{\prime}-s_{1}\right)\right)-\exp \left(-\delta_{1}\right)\right)^{+},
\end{aligned}
$$

where $(x)^{+}=\max (x, 0)$, and

$r_{0}=2 \sqrt{\alpha}(1-\sqrt{\alpha})\left|h_{a w}\right|^{2} P_{a} /\left(\sigma_{c w}^{2} P_{c}\right)$,

$s_{0}=\left(\sigma_{n}^{2}+(1-\alpha)\left|h_{a w}\right|^{2} P_{a}\right) /\left(\sigma_{c w}^{2} P_{c}\right)$,

$r_{1}=2(1-\sqrt{\alpha})\left|h_{a w}\right|^{2} P_{a} /\left(\sigma_{c w}^{2} P_{c}\right)$,

$s_{1}=\sigma_{n}^{2} /\left(\sigma_{a c}^{2} P_{c}\right)$,

$\delta_{0}=\left|h_{a w}\right|^{2} P_{a} /\left(\left(2^{R_{v}}-1\right) \sigma_{c w}^{2} P_{c}\right)-s_{1}$,

$\delta_{1}=\left|h_{a w}\right|^{2} P_{a}\left(1-(1-\alpha) 2^{R_{v}}\right)^{+} /\left(\left(2^{R_{v}}-1\right) \sigma_{c w}^{2} P_{c}\right)-s_{1}$.

Willie attempts to minimize $\left(P_{f}^{\prime}\left(h_{a w}\right)+P_{m}^{\prime}\left(h_{a w}\right)\right)$ by properly choosing $\lambda^{\prime}$ equal to

$$
\lambda_{*}^{\prime}=\min \left\{\frac{\ln \left(r_{1} / r_{0}\right)-\left(s_{0}-s_{1}\right)}{r_{1}-r_{0}}, \frac{s_{1}+\delta_{1}}{r_{1}}\right\},
$$

which its proof is provided in Appendix A, and then

$$
\begin{aligned}
P_{f}^{\prime}\left(h_{a w}\right)+P_{m}^{\prime}\left(h_{a w}\right)= & 1-\exp \left(s_{0}-r_{0} / \lambda_{*}^{\prime}\right) \\
& \left.+\exp \left(s_{1}-r_{1} / \lambda_{*}^{\prime}\right)\right)-\exp \left(-\delta_{1}\right) .
\end{aligned}
$$

\section{Average total detection error probability}

In summary, the average total detection error probability, estimated over the event of Willie's success and failure to decode $\mathbf{v}$, is given by

$$
\xi=\left(P_{f}+P_{m}\right)+\mathrm{E}_{h_{a w}}\left[P_{f}^{\prime}\left(h_{a w}\right)+P_{m}^{\prime}\left(h_{a w}\right)\right],
$$

which can be computed from (11) and (18).

Special case: When Willie perfectly knows his received AN power of $\left|h_{c w}\right|^{2} P_{C}$, he can choose the detection threshold $\lambda^{\prime} \in\left[\frac{2 \sqrt{\alpha}(1-\sqrt{\alpha})\left|h_{a w}\right|^{2} P_{a}}{\sigma_{n}^{2}+\left|h_{c w}\right|^{2} P_{c}+(1-\alpha)\left|h_{a w}\right|^{2} P_{a}}, \frac{2(1-\sqrt{\alpha})\left|h_{a w}\right|^{2} P_{a}}{\sigma_{n}^{2}+\left|h_{c w}\right|^{2} P_{c}}\right]$ such that the false alarm and missed detection probabilities are zero, i.e. $P_{f}^{\prime}\left(h_{a w}\right)=P_{m}^{\prime}\left(h_{a w}\right)=0$. Then, it follows from (11) and (19) that the average detection error probability reduces to

$$
\xi=1-\frac{\exp \left(-\left(2^{R_{v}}-1\right) \sigma_{n}^{2} /\left(\sigma_{a w}^{2} P_{a}\right)\right)}{1+\left(2^{R_{v}}-1\right) \sigma_{c w}^{2} P_{c} /\left(\sigma_{a w}^{2} P_{a}\right)} .
$$

\section{Covert Throughput}

The covert throughput defined by the maximum throughput 2 of the covert message $\mathbf{u}$ between Alice and Bob under constraint of the covert requirement, will be determined in this section. Since Alice does not know $h_{a b}$, he will transmit $\mathbf{u}$ at a fixed rate $R_{u}$. The received signal at Bob is given by

$$
\boldsymbol{y}_{b}=\sqrt{\alpha P_{a}} h_{a b} \mathbf{v}+\sqrt{(1-\alpha) P_{a}} h_{a c} \mathbf{u}+\sqrt{P_{c}} h_{c b} \mathbf{z}+\mathbf{n}_{b} .
$$

Assuming successive interference cancellation receiver type at Bob [16], thus the covert message maximum rate is given by

$$
I\left(\mathbf{u} ; \mathbf{y}_{b}\right)_{0}=\log _{2}\left(1+\frac{(1-\alpha)\left|h_{a b}\right|^{2} P_{a}}{\sigma_{n}^{2}+\alpha\left|h_{a b}\right|^{2} P_{a}+\left|h_{c b}\right|^{2} P_{b}}\right)
$$

if Bob cannot decode $\mathbf{v}$, i.e. $I\left(\mathbf{v} ; \mathbf{y}_{b}\right)<R_{v}$ or, equivalently,

$$
\left|h_{a b}\right|^{2}<\frac{\left(2^{R_{v}}-1\right)\left(\sigma_{n}^{2}+\left|h_{c b}\right|^{2} P_{b}\right)}{\left(1-(1-\alpha) 2^{R_{v}}\right) P_{a}},
$$

and, otherwise,

$$
I\left(\mathbf{u} ; \mathbf{y}_{b}\right)_{1}=\log _{2}\left(1+\frac{(1-\alpha)\left|h_{a b}\right|^{2} P_{a}}{\sigma_{n}^{2}+\left|h_{c b}\right|^{2} P_{b}}\right) .
$$

Then, the decoding outage probability, denoted $P_{\text {out }, B}\left(P_{a}\right)$, of the covert message $\mathbf{u}$ can be derived in (25).

Therefore, the covert throughput is given by

$$
\begin{array}{cl}
\eta_{u}=\max _{P_{a}, R_{u}} & R_{u} \times\left(1-P_{\text {out }, B}\left(P_{a}\right)\right) \\
\text { s.t. } & \xi \geq 1-\epsilon,
\end{array}
$$

where $\epsilon$ represents the covertness requirement. Since $\xi$ is a decreasing function of $P_{a}$, the constraint of $\xi \geq 1-\epsilon$ requires $P_{a}$ less than a threshold $P_{a}^{*}$. Also since $P_{\text {out }, B}\left(P_{a}\right)$ is an increasing function of $P_{a}, R_{u}\left(1-P_{\text {out }, B}\left(P_{a}\right)\right)$ is maximized when $P_{a}=P_{a}^{*}$. Therefore, the covert throughput is given by

$$
\eta_{u}=\max _{R_{u}} R_{u} \times\left(1-P_{\text {out }, B}\left(P_{a}^{*}\right)\right) .
$$

\footnotetext{
${ }^{2}$ The throughput of $\mathbf{u}$ between Alice and Bob is the average rate correctly received over many transmission bursts, i.e. $R_{u} \times\left(1-P_{\text {out }, B}\right)$, because the message is only correctly received on $\left(1-P_{\text {out }, B}\right)$ transmissions [16]
} 


$$
\begin{aligned}
& P_{\text {out }, B}\left(P_{a}\right)=\operatorname{Pr}\left(I\left(\mathbf{u} ; \mathbf{y}_{b}\right)_{0}<R_{u},\left|h_{a b}\right|^{2}<\frac{\left(2^{R_{v}}-1\right)\left(\sigma_{n}^{2}+\left|h_{c b}\right|^{2} P_{b}\right)}{\left(1-(1-\alpha) 2^{R_{v}}\right) P_{a}}\right)+\operatorname{Pr}\left(I\left(\mathbf{u} ; \mathbf{y}_{b}\right)_{1}<R_{u},\left|h_{a b}\right|^{2} \geq \frac{\left(2^{R} R_{v}-1\right)\left(\sigma_{n}^{2}+\left|h_{c b}\right|^{2} P_{b}\right)}{\left(1-(1-\alpha) 2^{R_{v}}\right) P_{a}}\right) \\
& =\operatorname{Pr}\left(\left|h_{a b}\right|^{2}<\min \left\{\frac{\left(2^{R_{v}}-1\right)\left(\sigma_{n}^{2}+\left|h_{c b}\right|^{2} P_{b}\right)}{\left(1-(1-\alpha) 2^{R_{v}}\right)+P_{a}}, \frac{\left(2^{R_{u}}-1\right)\left(\sigma_{n}^{2}+\left|h_{c b}\right|^{2} P_{b}\right)}{\left(1-\alpha 2^{R} u\right)+P_{a}}\right\}\right)+\operatorname{Pr}\left(\frac{\left(2^{R_{v}}-1\right)\left(\sigma_{n}^{2}+\left|h_{c b}\right|^{2} P_{b}\right)}{\left(1-(1-\alpha) 2^{R_{v}}\right)+P_{a}} \leq\left|h_{a b}\right|^{2} \leq \frac{\left(2^{R_{u}}-1\right)\left(\sigma_{n}^{2}+\left|h_{c b}\right|^{2} P_{b}\right)}{(1-\alpha) P_{a}}\right) \\
& =1-\max \left\{\frac{e^{-\frac{\left(2^{R} v-1\right) \sigma_{n}^{2}}{\left(1-(1-\alpha) 2^{R} v\right)+\sigma_{a b}^{2} P_{a}}}}{1+\frac{\left(2^{R} v-1\right) \sigma_{c b}^{2} P_{b}}{\left(1-(1-\alpha) 2^{R}\right)+\sigma_{a b}^{2} P_{a}}}, \frac{e^{-\frac{\left(2^{R} u-1\right) \sigma_{n}^{2}}{\left(1-\alpha 2^{R} u\right)+\sigma_{a b}^{2} P_{a}}}}{1+\frac{\left(2^{R} u-1\right) \sigma_{c b}^{2} P_{b}}{\left(1-\alpha 2^{R} u\right)+\sigma_{a b}^{2} P_{a}}}\right\}+\left(\frac{e^{-\frac{\left(2^{R} v-1\right) \sigma_{n}^{2}}{\left(1-(1-\alpha) 2^{R}\right)^{+} \sigma_{a b}^{2} P_{a}}}}{1+\frac{\left(2^{R}-1\right) \sigma_{c b}^{2} P_{b}}{\left(1-(1-\alpha) 2^{2} v\right)+\sigma_{a b}^{2} P_{a}}}-\frac{e^{-\frac{\left(2^{R} R_{u}-1\right) \sigma_{n}^{2}}{\sigma_{a b}^{2} P_{a}}}}{1+\frac{\left(2^{R} u-1\right) \sigma_{c b}^{2} P_{b}}{\sigma_{a b}^{2} P_{a}}}\right)^{+} .
\end{aligned}
$$

\section{Overt Throughrut Loss}

In this section, we characterize the loss of overt throughput traded for the covert throughput. When $\alpha=1$ (no transmission of covert message), the overt throughput, denoted $\eta_{v, n c}$, can be obtained from (4) and (5),

$$
\eta_{v, n c}=\max _{R_{v}} R_{v} \times \frac{\exp \left(-\left(2^{R_{v}}-1\right) \sigma_{n}^{2} /\left(P_{a}^{*} \sigma_{a c}^{2}\right)\right)}{1+\left(2^{R_{v}}-1\right) \phi P_{c} \sigma_{c c}^{2} /\left(P_{a}^{*} \sigma_{a c}^{2}\right)}
$$

Therefore, we obtain from (5) and (28) that the overt throughput loss is given by

$$
\eta_{v, l o s s}=\eta_{v, n c}-\eta_{v}
$$

which can be found by numerical search.

\section{NUMERICAL RESULTS}

The numerical results of the average detection error probability, $\xi$, and the covert throughput, $\eta_{u}$, are shown. For simplicity, we set $\sigma_{i j}^{2}=1$ for all $i, j, \sigma_{n}^{2}=1$ and $\alpha=0.8$.

Fig. 2 illustrates the average detection error probability versus $P_{a}$ for different values of the transmit AN power, $P_{c}$. It can be observed that $\xi$ decreases and converges to 0 as Alice's transmit power increases. It can also be observed that the average detection error probability significantly increases as the transmit AN power, $P_{c}$, increases even if Willie perfectly knows the AN power, and converges to 1 for high transmit AN power, as also shown in Fig. 3 of $\xi$ versus $P_{c}$ for different values of $\phi$. This indicates that the AN helps improve the covertness. Moreover in Fig. 3, the average detection error probability increases as the cancellation coefficient, $\phi$, decreases. This means that the improved performance of Carol's self-interference cancellation can also help improve the covertness.

Fig. 4 presents the covert throughput versus $\sigma_{c w}^{2}$ for different values of $\phi$. It can be observed that $\eta_{u}$ increases significantly as $\sigma_{c w}^{2}$ increases. Thus, the AN will be more effective when Willie is located closer to Bob. It can also be observed that the covert throughput increases as $\phi$ increases. The increase provided by the better performance of selfinterference cancellation is nearly constant. This indicates the positive impact of overt receiver's performance of selfinterference cancellation on the covert performance.

Fig. 5 shows the covert throughput and the overt throughput loss versus the transmit $\mathrm{AN}$ power, $P_{c}$, for different values of $\epsilon$. It can be observed that the covert throughput increases as the transmit $\mathrm{AN}$ power, $P_{c}$, increases and the increase is less significant for stricter covert requirements (smaller $\epsilon)$. However, to achieve a significant increase of the covert throughput by increasing AN's transmit power, it requires a trade of high overt throughput loss

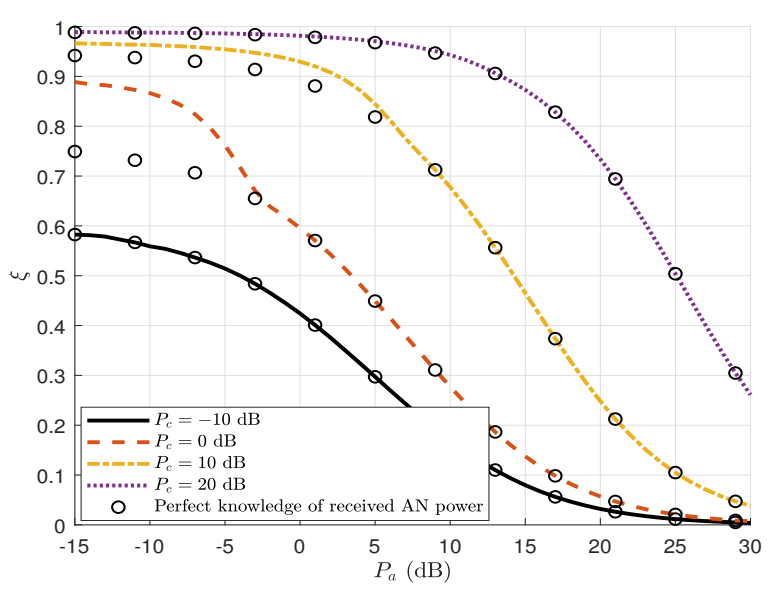

Fig. 2. The average detection error probability, $\xi$, versus Alice's transmit power, $P_{a}$, for different values of $P_{c}$.

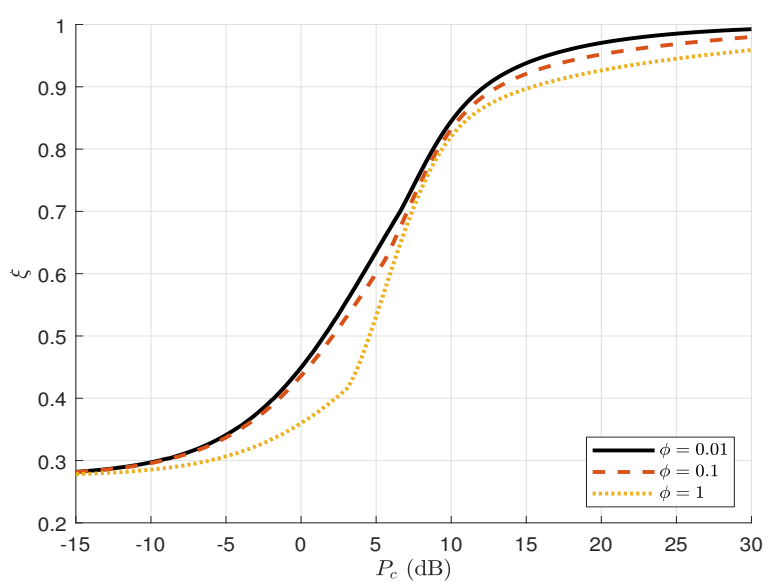

Fig. 3. The average detection error probability, $\xi$, versus Carol's transmit AN power, $P_{c}$, for different values of $\phi ; P_{a}=5 \mathrm{~dB}$.

\section{CONCLUSION}

This letter exploited the secure connection transmissions with a FD receiver to hide the covert information. Warden's uncertainties of decoding the overt message and AN received power are used to guarantee the covertness. We determined the average detection error probability and the covert throughput. The results showed that AN generated by the overt user can help improve the covertness and increase the maximum allowed transmit power, hence covert throughput. The covertness is further improved for the larger transmit power of $\mathrm{AN}$; however, it requires the trade of high overt throughput loss. 


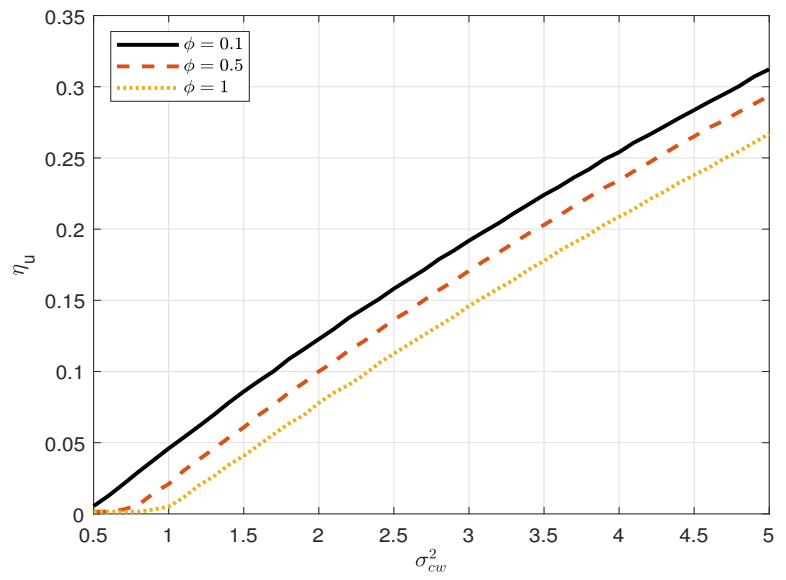

Fig. 4. The covert throughput, $\eta_{u}$, versus $\sigma_{c w}^{2}$, for different values of $\phi$; $\epsilon=0.1$ and $P_{c}=5 \mathrm{~dB}$.

The result also showed that the improved performance of selfinterference cancellation at the overt receiver can help improve the covertness, indicating the positive impact of the improved existing transmissions on the covert performance.

\section{ACKNOWLEDGMENT}

This study was carried out in close international collaboration between the School of Electrical Engineering, International University, Vietnam National University of Ho Chi Minh city and Lab-STICC, University of Brest, France. This research is supported by the Brest Institute of Computer Science and Mathematics (IBNM) CyberIoT Chair of Excellence of the University of Brest.

\section{APPENDIX A}

In this Appendix, we find the optimal detection threshold to minimize $\left(P_{f}^{\prime}\left(h_{a w}\right)+P_{m}^{\prime}\left(h_{a w}\right)\right)$ and its resulting minimum. Let $x=1 / \lambda^{\prime}$. Since $r_{1} \geq r_{0}, s_{0} \geq s_{1}, \delta_{0} \geq \delta_{1}$, it follows from (14) and (15) that

$$
\begin{aligned}
& P_{f}^{\prime}\left(h_{a w}\right)+P_{m}^{\prime}\left(h_{a w}\right) \\
= & \left\{\begin{array}{lr}
1-\exp \left(-\delta_{0}\right), & x \geq \frac{s_{0}+\delta_{0}}{r_{0}} \\
1-\exp \left(-\left(r_{0} x-s_{0}\right)\right), & \frac{s_{1}+\delta_{1}}{r_{1}} \leq x<\frac{s_{0}+\delta_{0}}{r_{0}}, \\
1-\exp \left(-\left(r_{0} x-s_{0}\right)\right) & x<\frac{s_{1}+\delta_{1}}{r_{1}} .
\end{array}\right.
\end{aligned}
$$

For $\left(s_{1}+\delta_{1}\right) / r_{1} \leq x<\left(s_{0}+\delta_{0}\right) / r_{0},\left(P_{f}^{\prime}\left(h_{a w}\right)+P_{m}^{\prime}\left(h_{a w}\right)\right)$ is increasing function of $x$ and hence, $\left(P_{f}^{\prime}\left(h_{a w}\right)+P_{m}^{\prime}\left(h_{a w}\right)\right)$ is minimized at $1-\exp \left(-r_{0}\left(s_{1}+\delta_{1}\right) / r_{1}+s_{0}\right)$. It follows from (30) that we can find optimum detection threshold and minimum of $1-\exp \left(s_{0}-r_{0} x\right)+\exp \left(s_{1}-r_{1} x\right)-\exp \left(-\delta_{1}\right)$ for $x<\left(s_{1}+\delta_{1}\right) / r_{1}$ by taking the first derivative and setting it to zero yield

$$
x=\min \left\{\left(\ln \left(r_{1} / r_{0}\right)-\left(s_{0}-s_{1}\right)\right) /\left(r_{1}-r_{0}\right),\left(s_{1}+\delta_{1}\right) / r_{1}\right\}
$$
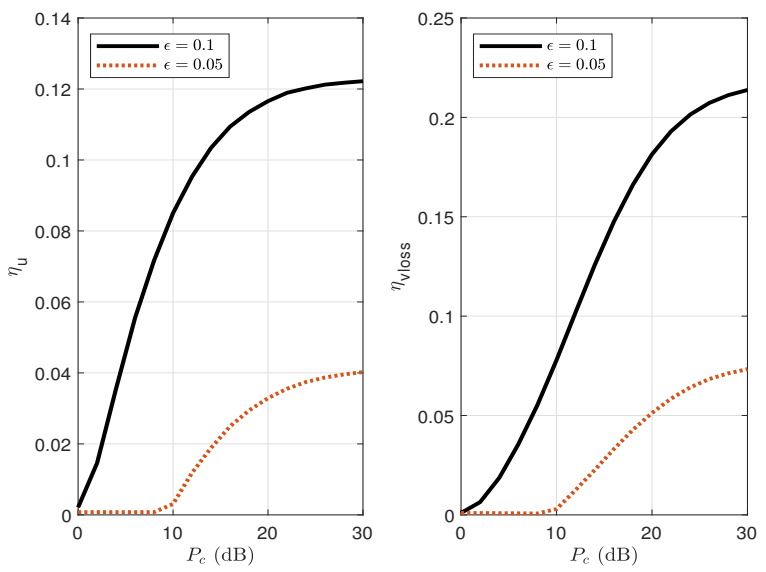

Fig. 5. $\eta_{u}$ and $\eta_{v, l o s s}$ versus the transmit AN power, $P_{c}$, for different values of $\epsilon ; \phi=0.1$.

\section{REFERENCES}

[1] J. Talbot and D.Welsh, Complexity and Cryptograhpy: An Introduction, Cambrige University Press, 2006.

[2] M. Bloch, J. Barros, M. R. Rodrigues, and S. W. McLaughlin, "Wireless information-theoretic security," IEEE Transactions on Information Theory, vol. 54, no. 6, pp. 2515-2534, 2008.

[3] S. Lee, R. J. Baxley, M. A. Weitnauer, and B. Walkenhorst, "Achieving undetectable communication," IEEE Journal of Selected Topics in Signal Processing, vol. 9, pp. 1195-1205, Oct 2015.

[4] D. Goeckel, B. Bash, S. Guha, and D. Towsley, "Covert communications when the warden does not know the background noise power," IEEE Communications Letters, vol. 20, pp. 236-239, Feb 2016.

[5] K. Shahzad, X. Zhou, S. Yan, J. Hu, F. Shu, and J. Li, "Achieving covert wireless communications using a full-duplex receiver," IEEE Transactions on Wireless Communications, vol. 17, no. 12, pp. 85178530, 2018.

[6] F. Shu, T. Xu, J. Hu, and S. Yan, "Delay-constrained covert communications with a full-duplex receiver," IEEE Wireless Communications Letters, vol. 8, no. 3, pp. 813-816, 2019.

[7] B. A. Bash, D. Goeckel, and D. Towsley, "Covert communication gains from adversarys ignorance of transmission time," IEEE Transactions on Wireless Communications, vol. 15, no. 12, pp. 8394-8405, 2016.

[8] H. Q. Ta and S. W. Kim, "Covert communication under channel uncertainty and noise uncertainty," in IEEE International Conference on Communications (ICC), pp. 1-6, 2019.

[9] S. Yan, B. He, X. Zhou, Y. Cong, and A. L. Swindlehurst, "Delayintolerant covert communications with either fixed or random transmit power," IEEE Transactions on Information Forensics and Security, vol. 14, no. 1, pp. 129-140, 2018.

[10] S. W. Kim and H. Q. Ta, "Covert communication by exploiting node multiplicity and channel variations," in IEEE International Conference on Communications (ICC), pp. 1-6, 2020.

[11] J. Hu, S. Yan, X. Zhou, F. Shu, J. Li, and J. Wang, "Covert communication achieved by a greedy relay in wireless networks," IEEE Transactions on Wireless Communications, vol. 17, no. 7, pp. 4766-4779, 2018.

[12] L. Tao, W. Yang, S. Yan, D. Wu, X. Guan, and D. Chen, "Covert communication in downlink NOMA systems with random transmit power," IEEE Wireless Communications Letters, vol. 9, no. 11, pp. 20002004, 2020.

[13] K. Shahzad, X. Zhou, S. Yan, J. Hu, F. Shu, and J. Li, "Achieving covert wireless communications using a full-duplex receiver," IEEE Transactions on Wireless Communications, vol. 17, no. 12, pp. 85178530, 2018.

[14] E. Everett, A. Sahai, and A. Sabharwal, "Passive self-interference suppression for full-duplex infrastructure nodes," IEEE Transactions on Wireless Communications, vol. 13, no. 2, pp. 680-694, 2014.

[15] E. Axell, G. Leus, E. G. Larsson, and H. V. Poor, "Spectrum sensing for cognitive radio: State-of-the-art and recent advances," IEEE signal processing magazine, vol. 29, no. 3, pp. 101-116, 2012.

[16] A. Goldsmith, Wireless communications. Cambridge Uni. press, 2005.

and then, the resulting minimum of $\left(P_{f}^{\prime}\left(h_{a w}\right)+P_{m}^{\prime}\left(h_{a w}\right)\right)$. 